\title{
Combination Therapy for Merkel Cell Carcinoma
}

\author{
Satadal Barik*
}

Department of Microbiology, Kumar Bhaskar Varma Sanskrit and Ancient Studies University, India

Corresponding author: Satadal Barik, Department of Microbiology, Kumar Bhaskar Varma Sanskrit and Ancient Studies University, Namati, Hati Namati- 781337, Nalbari, Assam, India, E-mail: satadalbarik1@gmail.com

Rec Date: Jun 21, 2016; Acc Date: Jul 18, 2016; Pub Date: Jul 25, 2016

Copyright: (c) 2016 Barik S. This is an open-access article distributed under the terms of the Creative Commons Attribution License, which permits unrestricted use, distribution, and reproduction in any medium, provided the original author and source are credited.

Citation: Barik S. Combination therapy for Merkel cell carcinoma. J Biomedical Sci. 2016, 5:4.

Keywords: Combination therapy; Idelalisib; NVP-BEZ235; Merkel cell carcinoma

\section{Merkel Cell Carcinoma}

Merkel Cell Carcinoma (MCC) is a lethal, aggressive uncommon neuroendocrine skin tumour that mainly occurs in the elderly, and there is no effective treatment. The presence of the Merkel-cell polyomavirus does not influence the activation of phosphoinositide 3-kinase (PI3K) in Merkel-Cell Carcinoma [1]. Both Merkel-cell polyomavirus-negative tumor tissues and tumor cells showed high expression of PI3Kס. A primary Merkelcell carcinoma cell line derived from tumors in the patient's lymph nodes. The PI3K signal transduction pathway is commonly activated in human cancers and regulates many of the hallmarks of cancer. The PI3K/AKT pathway played a vital role and is activated in the majority of human MCCs, and the PI3K inhibitor LY-294002 demonstrated a high sensitivity to MCC cell lines [2]. Idelalisib, a selective inhibitor of the delta isoform of PI3K (PI3K $)$, provides impressive therapeutic efficacy to treat patients with certain B-cell hematologic cancers [3].

In this issue of the Journal, a clinical report suggests that a complete clinical response induced by idelalisib in an 86-year-old white woman with stage IV Merkel-cell carcinoma of the right temple. Initially she underwent several surgery and radiation therapy leading to the multiple mutations, including a PIK3CA mutation $(c .1412 C \rightarrow T$ ). Furthermore, she underwent palliative hypofractionated radiation therapy. But all these attempts were failed and metastatic disease was confirmed by a liver biopsy. A shrinkage of liver lesion was visualised after one week of treatment with idelalisib and there was no visible tumor in her liver 3 months later analyse on positron-emission tomography and computed tomography (PET-CT), that suggesting a complete clinical response to idelalisib without significant adverse effects [4]. The therapeutic intervention involving PI3K pathway that heralds a new era of targeted therapy for the treatment of stage IV Merkel-cell carcinoma. Moreover, idelalisib suppressed tumor-cell growth in patient and induced cell death in vitro. In this study, the patient received hypofractionated radiation to the liver shortly before the idelalisib treatment. So, it fosters the hypothesis that hypofractionated radiation was partially responsible for the remission observed in this case study. However Shiver et al. reported that hypofractionated radiation therapy alone has not eradicated any visceral metastases in patients with Merkel-cell carcinoma. Although a synergistic effect might play a crucial role for this therapy, idelalisib played a prime role in eliminating the liver tumor in patient. Recent studies suggest that inhibition of PI3K $\delta$ perturbs B-cell signalling. It also suppresses the regulatory $T$ cells and unleashes CD8(+) cytotoxic $T$ cells that results the transfer of the balance from immune tolerance toward effective antitumor immunity. This phenomenon provides a rationale for the consideration of PI3K $\delta$ inhibitors as a therapeutic platform for wider use in solid tumors [5]. Many patients develop gastrointestinal symptoms during idelalisib therapy. Fifty patients were identified and treated with idealisib for 3 months, among them twenty-three (46\%) patients experienced diarrhea, twelve patients had colitis characterized by the combination of intraepithelial lymphocytosis and crypt cell apoptosis. B-cell neoplasia patients commonly express diarrheal symptoms when treated with idealisib. Patients who received dose-escalated idelalisib therapy can experience severe diarrhea or colitis, hepatotoxicity, pneumonitis and intestinal perforation as a consequence of the treatment-emergent adverse events (TEAEs) $[6,7]$.

$\mathrm{PI3K} /$ mammalian target of rapamycin (mTOR) pathway is found in numerous types of cancer, and play a key role in controlling cell proliferation, apoptosis and angiogenesis. NVPBEZ235 is an orally bioavailable imidazo(4,5-c)quinoline derivative has been reported to inhibit tumor proliferation and induces cell cycle arrest in Merkel cell carcinoma MKL-1 cells. The potential utility of NVP-BEZ235 is already established to inhibit a dual PI3K/mTOR pathway in MCC cell line. Furthermore, the levels of the cell cycle inhibitors p21 and p27 were upregulated in this class of drug treatment. These results possess considerable implications of NVP-BEZ235 as a potential therapeutic agent for the treatment of MCC [8]. Combination therapy with idelalisib is presumed to have a satisfactory safety profile. From last few years, the exciting progresses in the treatment of MCC open up a thrilling era for the treatment of MCC and associated disorders. Combining the novel agents in a logical way can perform in a prolific way to produce the maximum benefit. Combination of idelalisib and rituximab showed a clinically significant activity with an acceptable toxicity profile in patients with relapsed chronic lymphocytic leukemia (CLL) who were less able to undergo chemotherapy. The combination of idelalisib and rituximab may be a treatment 
option for the patients with CLL and can considerably improve progression-free survival and response rate among patients [3]. Targeting PI3K $\delta$ pathway with idelalisib is a significant first step approach that can be acquired for the therapeutic strategy in MCC. Idelalisib acts as a dose dependant manner and dose escalation may cause several disorders including diarrhea, hepatotoxicity and pneumonitis in patients [7]. Despite a reduced level of dose, idelalisib might act in a prolific way in combination with other effective agents with acceptable side effects. PI3K $\delta$ inhibitor (idelalisib) used in combination with NVP-BEZ235 provide promises to radically alter the attitude for the treatment of MCC and might lead to an effective therapeutic strategy with less toxicity. This approach appears likely to herald the beginning of a revolution in the treatment of MCC. Additional studies will be required to define the most successful use of these new agents.

\section{References:}

1. Nardi V, Song Y, Santamaria-Barria JA, Cosper AK, Lam Q, et al. (2012) Activation of PI3K signaling in Merkel cell carcinoma. Clin Cancer Res 18: 1227-1236.

2. Hafner C, Houben R, Baeurle A, Ritter C, Schrama D, et al. (2012) Activation of the PI3K/AKT pathway in Merkel cell carcinoma. PLoS One 7: e31255.
3. Furman RR, Sharman JP, Coutre SE, Cheson BD, Pagel JM, et al. (2014) Idelalisib and rituximab in relapsed chronic lymphocytic leukemia. N Engl J Med 370: 997-1007.

4. Shiver MB, Mahmoud F, Gao L (2015) Response to Idelalisib in a Patient with Stage IV Merkel-Cell Carcinoma. N Engl J Med 373: 1580-1582.

5. Ali K, Soond DR, Pineiro R, Hagemann T, Pearce W, et al. (2014) Inactivation of $\mathrm{PI}(3) \mathrm{K}$ p110 $\delta$ breaks regulatory T-cell-mediated immune tolerance to cancer. Nature 510: 407-411.

6. Weidner AS, Panarelli NC, Geyer JT, Bhavsar EB, Furman RR, et al. (2015) Idelalisib-associated Colitis: Histologic Findings in 14 Patients. Am J Surg Pathol 39: 1661-1667.

7. Coutré SE, Barrientos JC, Brown JR, de Vos S, Furman RR, et al. (2015) Management of adverse events associated with idelalisib treatment: expert panel opinion. Leuk Lymphoma 56: 2779-2786.

8. Lin Z, Mei H, Fan J, Yin Z, Wu G (2015) Effect of the dual phosphatidylinositol 3-kinase/mammalian target of rapamycin inhibitor NVP-BEZ235 against human Merkel cell carcinoma MKL-1 cells. Oncol Lett 10: 3663-3667. 